\title{
Effect of low-FODMAP diet in Finnish IBS patients. Results from non-controlled 12-week intervention study
}

\author{
$\underline{\text { Reijo Laatikainen }}^{1}$, Tuija Poussa ${ }^{2}$, Jari Koskenpato ${ }^{3}$, Markku Hillilä $^{4}$, Jussi Loponen ${ }^{5}$, \\ Sanna-Maria Hongisto ${ }^{5}$ and Riitta Korpela ${ }^{6}$ \\ ${ }^{1}$ Booston Ltd, Helsinki, Finland, \\ ${ }^{2}$ Stat consulting ltd, Nokia, Finland, \\ ${ }^{3}$ Aava Medical Centre, Helsinki, Finland, \\ ${ }^{4}$ HUS, Helsinki, Finland, \\ ${ }^{5}$ Fazer Oyj, Vantaa, Finland and \\ ${ }^{6}$ University of Helsinki, Helsinki, Finland
}

\begin{abstract}
Introduction

Low FODMAP diet is shown to alleviate symptoms of irritable bowel syndrome in many populations. Until today, no holistic FODMAP diet studies have been published among Finnish IBS patients.

Materials and Methods

This study was a 12-week non-controlled follow-up study of a previously published randomised cross-over trial on different rye bread products ${ }^{1}$. In this follow-up study $(\mathrm{n}=50)$ symptom values during rye bread periods, i.e. low-FODMAP and regular rye periods, are used as baseline values.

Participants were instructed to follow holistic low-FODMAP diet after they had first consumed regular and low-FODMAP rye bread for 4 weeks of each bread. Symptoms were measured with a validated symptom severity scoring system (IBS-SSS. Quality of life (QOL) was monitored and fiber intake during the rye bread periods and low-FODMAP diet was calculated from 4-day
\end{abstract} food diaries.

\section{Results}

Mean IBS-SSS was 211 (SD 84) during low-FODMAP rye bread and 218 (86) during regular rye bread. During low-FODMAP diet IBS-SSS was 178 (79) at week 1 and reduced further to $135(80)$ by the end of week $12(\mathrm{p}<0.001)$. IBS-SSS scores at week 1 and week 12 during low-FODMAP diet were lower compared to low-FODMAP rye bread and regular rye bread ( $p<0.01$ for all comparisons.

Mean QOL was 68 during low-FODMAP rye bread, 67 during regular rye bread; 68 at week 1 and 74 at week 12 during low-FODMAP diet. At week 1 no improvement was found in QOL vs. either rye bread period, but at week 12 the improvement was significant ( $p<0.001$ vs. both rye breads).

Fiber intake was lower at the end of week 12 (median $19 \mathrm{~g} / \mathrm{d})$ than during either low-FODMAP rye bread (28 g/d) or regular rye bread period $(31 \mathrm{~g})(\mathrm{p}<0.001$ vs. both rye breads).

\section{Discussion}

Low-FODMAP diet reduced the symptoms of IBS and improved quality of life in Finnish IBS patients. However, due to noncontrolled nature of this follow-up study, the results need to be interpreted with caution, and randomised studies are warranted also in Finland. Achieving recommended intake of fiber seems to be challenging for Finnish patients with IBS, and therefore the use high fiber food items and/or fiber supplements which are also low in FODMAP should be encouraged.

\section{Conflict of Interest}

RL has written a Finnish book on irritable bowel syndrome and diet. He is also the founder and owner of Booston Ltd, which provides IBS-related dietetic services to IBS patients, healthcare professionals and various organisations. S-MH and JL are employees of Fazer Group. Other authors do not report any competing interests. 